Pacific Journal of Mathematics

AN APPROXIMATION THEOREM FOR THE POISSON 


\title{
AN APPROXIMATION THEOREM FOR THE POISSON BINOMIAL DISTRIBUTION
}

\author{
LUCIEN LE CAM
}

1. Introduction. Let $x_{j} ; j=1,2, \cdots$ be independent random variables such that $\operatorname{Prob}\left(X_{j}=1\right)=1-\operatorname{Prob}\left(X_{j}=0\right)=p_{j}$. Let $Q=\mathscr{L}\left(\Sigma X_{j}\right)$ be the distribution of their sum. This kind of distribution is often referred to as a Poisson binomial distribution. For any finite measure $\mu$ on the real line let $\|\mu\|$ be the norm defined by

$$
\|\mu\|=\sup _{f}\left\{\left|\int f d \mu\right|\right\} .
$$

the supremum being taken over all measurable functions $f$ such that $|f| \leqq 1$. Let $\lambda=\Sigma p_{j}$, let $\Sigma p_{j}^{2}=\lambda \approx$ and let $\alpha=\sup _{j} p_{j}$. Finally let $P$ be the Poisson distribution whose expectation is equal to $\lambda$.

The purpose of the present paper is to show that there exist absolute constants $D_{1}$ and $D_{2}$ such that $\|Q-P\| \leqq D_{1} \alpha$ for all values of the $p_{j}^{\prime}$ 's and $\|Q-P\| \leqq D_{2} \approx$ if $4 \alpha \leqq 1$.

The constant $D_{1}$ is not larger than 9 and the constant $D_{2}$ is not larger than 16.

Such a result can be considered a generalization of a theorem of Yu. V. Prohorov [9] according to which such constants exist when all the probabilities $p_{j}$ are equal.

The norm $\|Q-P\|$ is always larger than the maximum distance $\rho(P, Q)$ between the cumulative distributions. For this distance $\rho$ a very general theorem of A. N. Kolmogorov [6] implies that $\rho(P, Q)$ is at most of order $\alpha^{1 / 5}$. The improvement obtained here is made possible by the smaller scope of our assumptions.

The method of proof used in the present paper is not quite elementary, since it uses both operator theoretic methods and characteristic functions. The relevant concepts are described in $\S 2$.

A completely elementary approach, described in [4] leads to bounds of the order of $3 \alpha^{1 / 3}$ for the distance $\rho$. Unfortunately, the elementary method does not seem to be able to provide the more precise result of the present paper.

The developments given here were prompted by discussions with J. H. Hodges, Jr. in connection with the writing of [4].

2. Measures as operators. Let $\{\mathfrak{C}, \mathfrak{A}\}$ be a measurable Abelian group, that is, an Abelian group on which a $\sigma$-field $\mathfrak{A}$ has been selected

Received December 9, 1959. The author is a research fellow of the A.P. Sloan Foundation. 
in such a way that the map $(x, y) \rightarrow x+y$ from $\mathfrak{X} \times \mathfrak{X}$ to $\mathfrak{X}$ is measurable for the $\sigma$-fields $\mathfrak{A} \times \mathfrak{A}$ and $\mathfrak{A}$.

Let $\mathscr{B}$ denote the set of bounded measurable numerical functions on $\{\mathfrak{X}, \mathfrak{A}\}$. A finite signed measure $\mu$ on $\mathfrak{A}$ defines an operator, also denoted $\mu$, from $\mathscr{B}$ to itself. To the function $f \in \mathscr{B}$ the operator $\mu$ makes correspond the element $\mu f$ whose value at the point $x$ is $(\mu f)(x)=$ $\int f(x+\xi) \mu(d \xi)$. Linear combinations of two operators are defined by the equality

$$
(\alpha \mu+\beta \nu) f=\alpha(\mu f)+\beta(\nu f) .
$$

The product of two operators will be defined by composition: $(\mu \nu) f=$ $\mu(\nu f)$. In other words,

$$
[(\mu \nu) f](x)=\int \mu(d y) \int f(x+\xi+y) \nu(d \xi) .
$$

It follows from Fubini's theorem that $\mu \nu=\nu \mu$. The product $\mu \nu$ corresponds to the convolution of the two measures.

For any element $f$ of $\mathscr{B}$ let $|f|$ be the norm $|f|=\sup |f(x)|$. Define the operator norm $\|\mu\|$ by

$$
\|\mu\|=\sup \{|\mu f| ;|f| \leqq 1\} .
$$

The norm $\|\mu\|$ is equal to the total mass of $\mu$ considered as a measure. It is an immediate consequence of the operator representation of $\mu \nu$ that $\|\mu \nu\| \leqq\|\mu\|\|\nu\|$.

Let $\mathfrak{M}$ be the system of operators obtained from all the finite signed measures. What precedes can be summarized by saying that $\mathfrak{M}$ is a normed commutative algebra having for identity the operator $I$ which is the probability measure whose mass is entirely concentrated at the point $x=0$. It is not difficult to show that $\mathfrak{M}$ is complete for the norm, so that $\mathfrak{M}$ is in fact a real commutative Banach algebra.

Let $\varphi$ be a complex-valued function of a complex variable $z$. Suppose that for $|z|<a$, the function $\varphi$ has a convergent power series expansion. It is then possible to define $\varphi(A)$ for every $A \in \mathfrak{M}$ such that $\|A\|<a$ by simple formal substitution in the power series expansion of $\varphi$.

The entity $\varphi(A)$ is then of the form $\varphi(A)=B+i C$ where both $B$ and $C$ belong to $\mathfrak{M}$. Other possible definitions can be found in [3], [2], [8]. If $\hat{\mu}$ is the Fourier transform $\hat{\mu}(t)=\int e^{i t x} \mu(d x)$ of the measure $\mu$ then $\varphi(\mu)$ is the measure where the Fourier transform is $\varphi(\hat{\mu})$.

In most cases of statistical interest, the space $\mathfrak{X}$ is either the real line, or the additive group of integers, or the circle, or a Euclidean space. In those circumstances, as well as in the case where $\mathfrak{X}$ is an arbitrary Abelian locally compact group, we may replace $\mathscr{B}$ by the space 
of continuous functions which tend to zero at infinity without affecting any of the above properties.

Let $M$ be an arbitrary finite positive measure on $\mathfrak{X}$. Then $\exp (M)=$ $e^{M}=I+M+\cdots+(1 / k) ! M^{k}+\cdots$. It follows that $\exp [M-\|M\| I]=$ $\exp [-\|M\|] \exp (M)$ is always a probability measure.

If a random variable $X$ is equal to the origin of $\mathfrak{X}$ with probability $(1-p)$ the distribution $\mathscr{L}(X)$ can be written $\mathscr{L}(X)=I+p(M-1)$ where $M$ is a probability measure.

The following theorem, essentially due to Khintchin [5] and Doeblin [1] is concerned with the distribution $Q$ of a sum $\Sigma X_{j}$ of independent variables having distributions $G_{j}=I+p_{j}\left(M_{j}-I\right)$ where $M_{j}$ is a probability measure. The product $\Pi_{j} G_{j}$ is always convergent when $\lambda=$ $\sum_{j} p_{j}$ is finite. Conversely finiteness of $\lambda$ is necessary to the convergence of $\Pi_{j} G_{j}$ when $\mathfrak{X}$ is the additive group of integers. More generally, suppose that $\mathfrak{X}$ is the real line and that there exists an $\varepsilon>0$ such that $\lambda_{z}=\Sigma p_{j} M_{j}\left\{[-\varepsilon, \varepsilon]^{c}\right\}=\infty$. Then $\Pi_{j} G_{j}$ cannot be convergent. This follows for instance from a result of Paul Lévy [7] according to which any interval containing the sum $\Sigma X_{j}$ with probability $\alpha>0$ must have a length of the order of $\varepsilon \sqrt{\lambda_{\varepsilon}}$.

A refinement of Paul Lévy's theorem can be found in [6], Lemma 1. However, the finiteness of $\lambda$ is not generally necessary to the convergence of $\Pi_{j} G_{j}$. This is quite obvious if $\mathfrak{X}$ is the circle and $G_{1}$ is the Haar measure of the circle, but the condition is not even necessary on the line.

Theorem 1. Let $X_{j} ; j=1,2, \cdots$ be independent random variables taking their values in the measurable Abelian group $\mathfrak{X}$. Assume that $\mathscr{L}\left(X_{j}\right)=I+p_{j}\left(M_{j}-I\right)$ where $M_{j}$ is a probability measure and assume that $\lambda=\Sigma p_{j}<\infty$. Let $p_{j}=\lambda c_{j}$, let $\widetilde{\sigma}=\Sigma c_{j} p_{j}$ and finally let $M=\Sigma c_{j} M_{j}$. Then

$$
\|Q-P\| \leqq 2 \lambda \approx
$$

for $P=\exp [\lambda(M-I)]$.

Proof. The proof is essentially the same as the proof of Theorem 1 in [4], given there in terms of random variables. In terms of operators one can proceed as follows.

Let $F_{j}=\exp p_{j}\left(M_{j}-I\right)$ and let $R_{1}=\prod_{j \geq 2} G_{j}$. For $k>1$ let $R_{k}=$ $\left(\Pi_{j \leqq k-1} F_{j}\right)\left(\prod_{j \geqq k+1} G_{j}\right)$. Then $R_{k} F_{k}=R_{k+1} G_{k+1}$ so that

$$
\prod_{j} G_{j}-\prod_{j} F_{j}=\sum_{j} R_{j}\left(G_{j}-F_{j}\right) .
$$

Since $R_{j}$ is a probability measure, this implies 


$$
\left\|\prod_{j} G_{j}-\prod_{j} F_{j}\right\| \geqq \sum_{j}\left\|G_{j}-F_{j}\right\| \text {. }
$$

The difference $F_{j}-G_{j}$ can be written

$$
F_{j}-G_{j}=\left[e^{-p_{j}}-\left(1-p_{j}\right)\right] I+p_{j}\left(e^{-p_{j}}-1\right) M_{j}+\sum_{k=2}^{\infty} \frac{e^{-p_{j}}}{k !} p_{j}^{k} M_{j}^{k} .
$$

Hence $\left\|F_{j}-G_{j}\right\| \leqq 2 p_{j}\left(1-e^{-p_{j}}\right) \leqq 2 p_{j}^{2}$.

Noting that $\Pi_{j} F_{j}=\exp [\lambda(M-I)]$, this proves the desired result.

REMARK. The literature does not seem to contain any reference to the fact that Theorem 1 can be proved as in [4] and coupled with Lindeberg's proof of the normal approximation theorem to obtain a completely elementary proof of the general Central Limit theorem.

3. Sums of indicator variables and binomial distributions. In all the subsequent sections of this paper $\mathfrak{X}$ will be the additive group of integers and $\left\{X_{j} ; 1,2, \cdots\right\}$ will be a family of independent random variables such that $\operatorname{Prob}\left(X_{j}=1\right)=1-\operatorname{Prob}\left(X_{j}=0\right)=P_{j}$. The distribution $\mathscr{L}\left(X_{j}\right)$ can then be written either as $I+p_{j} \Delta$ or $\left(1-p_{j}\right) I+p_{j} H$ where $\Delta$ is the difference operator $\Delta=H-I$ and $H$ is the probability measure whose mass is entirely concentrated at the point $x=1$. The Poisson distribution whose expectation is $\lambda$ can be written $P=\exp (\lambda \Delta)$.

Letting $\lambda c_{j}=p_{j}$ and $\widetilde{\sigma}=\Sigma c_{j} p_{j}$, Theorem 1 implies that if $Q=$ $\mathscr{L}\left(\Sigma X_{j}\right)$ then the following inequality holds.

Proposition 1. $\|Q-\exp (\lambda \Delta)\| \leqq 2 \lambda \tau$.

From now on we shall assume that $\lambda<\infty$ and that $\alpha=\sup p_{j}$ does not exceed $1 / 4$.

It may be expected that $Q$ would be approximable by a binomial distribution much more closely than by a Poisson distribution. Letting $\lambda=\nu \pi$, a binomial distribution with $\nu$ trials and probability of success $\varpi$ can be written

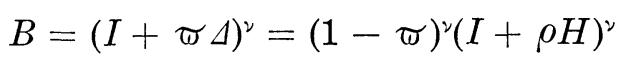

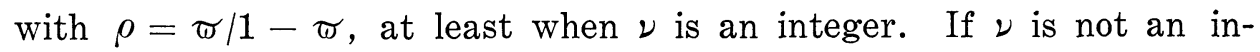
teger the expression

$$
B=(1-\tau)^{\nu}\left\{I+\left(\begin{array}{l}
\nu \\
1
\end{array}\right) \rho H+\cdots+\left(\begin{array}{l}
\nu \\
k
\end{array}\right) \rho^{k} H^{k}+\cdots\right\}
$$

where

$$
\left(\begin{array}{l}
\nu \\
k
\end{array}\right)=\frac{1}{k !} \nu(\nu-1) \cdots(\nu-k+1)=\frac{\Gamma(\nu+1)}{k ! \Gamma(\nu-k+1)}
$$


still possesses a precise meaning as long as $\rho<1$. However, $B$ is not a probability measure even though $\int 1 d B=1$. Let $n$ be the integer such that $(n-1)<\nu \leqq n$. The coefficients $\left(\begin{array}{l}\nu \\ k\end{array}\right)$ of order $k=(n+1),(n+2) \cdots$ are alternately positive and negative.

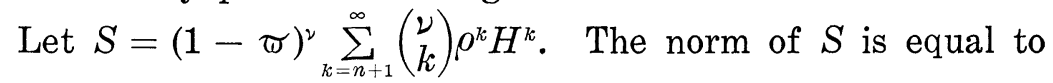

$$
\|S\|=(1-\widetilde{\sigma})^{\nu} \sum_{k=n+1}^{\infty}\left|\left(\begin{array}{l}
\nu \\
k
\end{array}\right)\right| \rho^{k}=(1-\widetilde{\sigma})\left|\sum_{k=n+1}^{\infty}\left(\begin{array}{l}
\nu \\
k
\end{array}\right)(-\rho)^{k}\right| .
$$

The term inside the absolute value symbol is simply the remainder of the expansion of $(1-\rho)^{2}$. By Taylor's formula $\|S\|$ is equal to the absolute value of

$$
\frac{1}{n !} \nu(\nu-1) \cdots(\nu-n)(1-\widetilde{\sigma})^{\nu}(1-\rho)^{\nu} \int_{0}^{\rho / 1-\rho}(-1)^{n} t^{n}(1+t)^{\nu-n-1} d t .
$$

Therefore, since $n-1<\nu<n$

$$
\begin{aligned}
& \|S\| \leqq(1-\widetilde{\sigma})^{\nu}(1-\rho)^{\nu} \int_{0}^{\rho / 1-\rho} t^{n}(1+t)^{-1} d t \\
& \leqq \frac{1}{n+1}(1-\widetilde{\sigma})^{\nu}(1-\rho)^{\nu}\left(\frac{\rho}{1-\rho}\right)^{n+1} \\
& =\frac{\widetilde{\sigma}^{n+1}}{n+1}(1-2 \widetilde{\sigma})^{\nu-n-1} \leqq \frac{4}{\nu+1} \widetilde{\varpi}^{\nu+1} \text {. }
\end{aligned}
$$

In the cases considered here $\nu=\left(\Sigma p_{j}\right)^{2}\left(\Sigma p_{j}^{2}\right)^{-1}$ is always larger than or equal to unity. In all cases where $\nu$ is large and $\widetilde{\sigma}$ is small $\|S\|$ will be rather negligible.

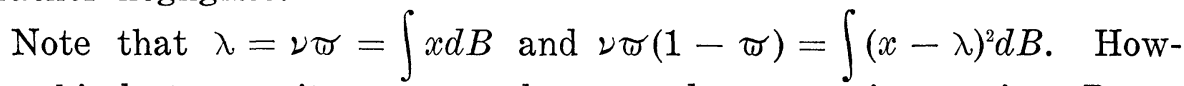
ever, this last quantity may not be treated as a variance, since $B$ possesses negative terms.

In spite of this it will be convenient to bound the remainder term

$$
S(m)=(1-\widetilde{\sigma})^{\nu} \sum_{k=m+1}^{\infty}\left(\begin{array}{l}
\nu \\
k
\end{array}\right) \rho^{k} H^{k}
$$

for large values of $m$, by Chebyshev's inequality. Assuming $\lambda<m \leqq n$

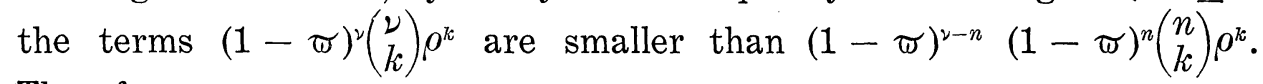
Therefore

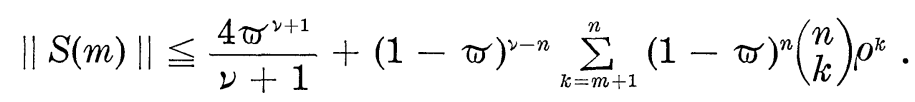

Finally, by Chebyshev's inequality applied to the binomial $[1+\widetilde{\sigma}]^{n}$, one obtains 


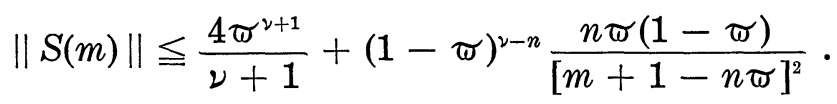

In particular, if $m \leqq 2 n \pi<m+1$

$$
\begin{aligned}
\|S(m)\| & \leqq \frac{4 \varpi^{\nu+1}}{\nu+1}+\frac{(1-\tau)^{1-(n-\nu)}}{n \varpi} \\
& \leqq\left[4 \varpi^{\nu+2}+1\right] \frac{1}{\lambda} .
\end{aligned}
$$

To show that $Q$ can be approximated by the Poisson distribution $P$ in the cases where $\lambda$ is too large for Proposition 1 to have any significance, we shall first show that $Q$ can be approximated by $B$ and then show that $B$ is very close to $P$. The argument will be divided into three parts according to the values of $\lambda$ and $\lambda a^{2}$ for $a^{2}=\Sigma c_{j}\left(p_{j}-\widetilde{\sigma}\right)^{2}$. If $\lambda$ is large but $\lambda a^{2}$ is small, bounds will be obtained through operator theoretic methods. If $\lambda$ is so large that $\lambda \alpha^{2}$ becomes large, bounds will be obtained through computations on characteristic functions.

4. Approximations by binomial distributions. In this section, it will be assumed throughout that $\lambda \geqq 3$ and that $\alpha \leqq 1 / 4$.

For the distributions $Q$ and $B$ defined in the preceding section we can write

$$
\begin{aligned}
& \log Q-\log B=\sum_{j} \log \left(I+p_{j} \Delta\right)-\nu \log (I+\widetilde{\sigma} \Delta) \\
& =\lambda \sum_{j} c_{j}\left\{\frac{1}{p_{j}} \log \left(I+p_{j} \Delta\right)-\frac{1}{\widetilde{\sigma}} \log (I+\widetilde{\sigma} \Delta)\right\} \\
& =\lambda \sum_{k=2}^{\infty} \frac{(-1)^{k}}{k+1} \beta_{k} \Delta^{k+1}=\lambda \Delta M \text {, }
\end{aligned}
$$

with

$$
M=\sum_{k=2}^{\infty} \frac{(-1)^{k}}{k+1} \beta_{k} \Delta^{k}
$$

and $\beta_{k}=\sum_{j} c_{j} p_{j}^{k}-\widetilde{\varpi}^{k} \geqq 0$.

Since $(-1)^{k} \Delta^{k}=\sum_{s=0}^{\infty}\left(\begin{array}{l}k \\ s\end{array}\right)(-1)^{s} H^{s}$, the measure $M$ assigns negative masses to the odd positive integers and positive masses to the even nonnegative integers.

The norm of $M$ is precisely equal to

$$
\|M\|=\sum_{k=2}^{\infty} \frac{\beta_{k} 2^{k}}{k+1}=-\sum_{j} c_{j}\left\{\frac{1}{2 p_{j}} \log \left(1-2 p_{j}\right)-\frac{1}{2 \pi} \log (1-2 \widetilde{\sigma})\right\} .
$$

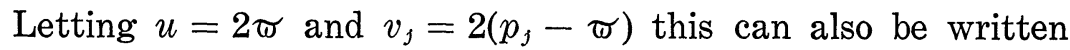




$$
\|M\|=\int_{0}^{1} \Sigma c_{j}\left\{\left[\frac{1}{1-t\left(u+v_{j}\right)}-\frac{1}{1-t u}\right]\right\} d t
$$

Since $\Sigma c_{j} v_{j}=0$ and $\Sigma c_{j} v_{j}^{2}=4 a^{2}$ while

$$
\left[1-t\left(u+v_{j}\right)\right]^{-1}-(1-t u)^{-1}=(1-t u)^{2}\left\{1+\left(t v_{j}\right)\left[1-t\left(u+v_{j}\right)\right]^{-1}\right\} t v_{j}
$$

one can write

$$
\begin{aligned}
\|M\| & =\int_{0}^{1}\left\{\Sigma c_{j} \frac{v_{j}^{2}}{\left[1-t\left(u+v_{j}\right)\right]}\right\} \frac{t^{2}}{(1-t u)^{2}} d t \\
& \leqq \frac{4 a^{2}}{1-2 \alpha} \int_{0}^{1} \frac{t^{2}}{(1-t u)^{2}} d t \\
& \leqq \frac{4 a^{2}}{3(1-2 \alpha)}\left\{1+\frac{3 \pi}{(1-2 \pi)^{2}}\right\} .
\end{aligned}
$$

Hence $\|M\|=h \alpha^{2}$ with

$$
h \leqq \frac{4}{3(1-2 \alpha)}\left\{1+\frac{3 \pi}{(1-2 \pi)^{2}}\right\} .
$$

One can also write $M=\Delta M_{1}=\Delta^{2} M_{2}$ with $\|M\|=2\left\|M_{1}\right\|=4\left\|M_{2}\right\|$.

It results from these equalities that

$$
Q=B \exp [\lambda \Delta M] \text {. }
$$

For every measure $\mu$, Taylor's formula gives

$$
e^{\mu}=I+\mu \int_{0}^{1} e^{\xi \mu} d \xi
$$

Hence

$$
\begin{aligned}
Q-B & =\lambda \Delta B M \int_{0}^{1} e^{\xi \lambda \Delta M} d \xi \\
& =\lambda \Delta^{2} B M_{1} \int_{0}^{1} e^{\xi \lambda \Delta M} d \xi .
\end{aligned}
$$

Finally

$$
\|Q-B\| \leqq \lambda\|M\|\|\Delta B\| e^{2 h \lambda a^{2}}
$$

and

$$
\|Q-B\| \leqq \frac{1}{2} \lambda\|M\|\left\|\Delta^{2} B\right\| e^{2 h \lambda a^{2}} .
$$

One can also note that there exist probability measures $F$ and $G$ such that if $\varepsilon=\|M\|$ then

$$
Q \exp [\lambda \varepsilon(F-I)]=B \exp [\lambda \varepsilon(G-I)] .
$$


According to the foregoing expressions, to obtain bounds on $\|Q-B\|$ it will be sufficient to evaluate $\|\Delta B\|$ and $\left\|\Delta^{2} B\right\|$.

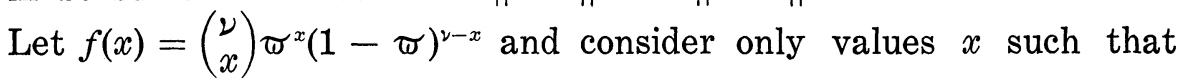
$x \leqq n-1$. In this range $f$ achieves its maximum at a value $x$ such that

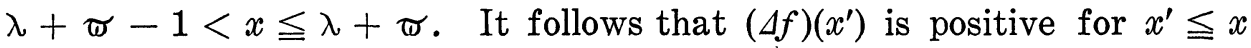
and negative for $x^{\prime}>x$. Finally

$$
\|\Delta B\| \leqq 2 f(x)+\|S\| \text {. }
$$

Let $x=\nu \xi$. An application of Stirling's formula leads to the inequality

$$
\begin{aligned}
\log f(x) & \leqq-\frac{1}{2} \log [2 \pi \nu \xi(1-\xi)] \\
f(x) & \leqq \frac{\theta}{\sqrt{\bar{\lambda}}}
\end{aligned}
$$

with

$$
\theta=\frac{1}{\sqrt{2 \pi}}\left[\frac{\xi}{\widetilde{\sigma}}(1-\xi)\right]^{-1 / 2}
$$

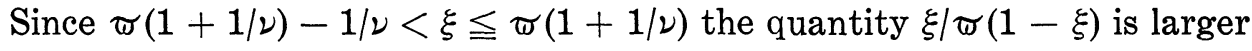
than

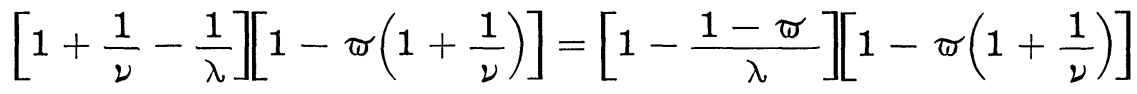

$$
\begin{aligned}
& \left.\geqq\left(1-\frac{1}{\lambda}\right)\left[1-\varpi\left(1+\frac{\varpi}{\lambda}\right)\right] \geqq \frac{2}{3}^{\prime} 1-\frac{13}{48}\right) \text {. }
\end{aligned}
$$

Consequently,

$$
\theta \leqq\left(\frac{72}{70 \pi}\right)^{1 / 2}
$$

and

$$
\|\Delta B\| \leqq \frac{2 \theta}{\sqrt{\lambda}}+\frac{4 \sigma^{\nu+2}}{\lambda}
$$

Thus, we have shown the validity of the following proposition.

Proposition 2. Let $\lambda \geqq 3$ and $\alpha \leqq 1 / 4$, then

$$
\|Q-B\| \leqq 2 h a^{2} \sqrt{\lambda} \exp \left(2 h \lambda a^{2}\right)\left\{\theta+\frac{4 \widetilde{\omega}^{2+2}}{\sqrt{\bar{\lambda}}}\right\}
$$

with 


$$
h \leqq \frac{4}{3}\left(\frac{1}{1-2 \alpha}\right)\left[1+\frac{3 \varpi}{(1-2 \varpi)^{2}}\right] \leqq \frac{32}{3}
$$

and

$$
\theta \leqq\left(\frac{36}{35 \pi}\right)^{1 / 2} \leqq \frac{1}{\sqrt{3}}
$$

A computation using the fact that $\Delta M=\Delta^{2} M_{1}$ and the bounds for $\left\|\Delta^{2} B\right\|$ can be carried out as follows.

Let $u=x+1-\nu \pi$ and let $f(u)$ be the probability of $x=$

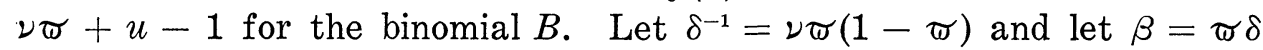
and $\gamma=(1-\widetilde{\sigma}) \delta$. Then

$$
\frac{f(u+1)}{f(u)}=\frac{1-\beta(u-1)}{1+\gamma u} .
$$

The second differences of the function $f$ for $x \leqq n$ are equal to some positive quantity multiplied by

$$
g(u)=u^{2}-(2 \varpi-1) u-(\nu+2) \varpi(1-\varpi) .
$$

Let $r_{1}$ and $r_{2}, r_{1}<r_{2}$ be the roots of this polynomial. The second differences $\left(\Delta^{2} f\right)(u)$ are negative for $u \in\left(r_{1}, r_{2}\right)$ and positive otherwise. Letting $\varphi(u)=(\Delta f)(u)$ it follows that

$$
\begin{aligned}
& \left\|\Delta^{2} B\right\| \leqq \varphi\left(u_{1}\right)+\left|\varphi\left(u_{2}\right)-\varphi\left(u_{1}-1\right)\right|+\varphi(n-\lambda+1)-\varphi\left(u_{2}-1\right) \\
& +\frac{8}{\nu+1} \widetilde{\omega}^{\nu+1}
\end{aligned}
$$

The values $u_{i}$ are determined by the condition that the corresponding $x$ values, say $x_{1}$ and $x_{2}$, are respectively the largest integer not exceeding $r_{1}+\lambda$ and the smallest integer as large as $r_{2}+\lambda$. The roots $r_{1}$ and $r_{2}$ are given by the expression

$$
r=(\varpi-1 / 2) \pm\left[(\nu+1) \omega(1-\omega)+\frac{1}{4}\right]^{1 / 2} .
$$

If $\lambda \geqq 3$ the value $u_{1}$ is negative while $u_{2}-1$ is positive.

In this case

$$
\begin{aligned}
\varphi\left(u_{1}\right) & \leqq f\left(u_{1}+1\right)\left[1-\frac{1+\gamma u_{1}}{1-\beta\left(u_{1}-1\right)}\right] \\
& \leqq f\left(u_{1}+1\right) \delta\left[\left|u_{1}\right|+\varpi\right] \\
& \leqq \frac{\theta}{\sqrt{\lambda}}|| u_{1}|+\widetilde{\varpi}| \frac{1}{\lambda(1-\varpi)} .
\end{aligned}
$$


Similarly,

$$
\begin{aligned}
& \left|\varphi\left(u_{2}\right)\right| \leqq f\left(u_{2}\right)\left[\frac{1-\beta\left(u_{2}-1\right)}{1+\gamma u_{2}}-1\right]
\end{aligned}
$$

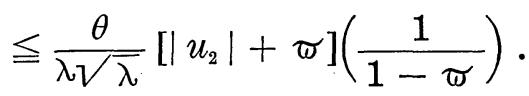

Note that $\left|u_{1}-1\right| \leqq 1+1 / 2+\sqrt{\nu \widetilde{\sigma}(1-\widetilde{\sigma})}+1 / 6 \leqq 5 / 3+\sqrt{\lambda(1-\widetilde{\sigma})}$. Hence

$$
\begin{aligned}
\varphi\left(u_{1}-1\right) & \leqq \frac{\theta}{\lambda}\left\{\frac{1}{(1-\widetilde{\sigma}) \sqrt{\lambda}}\left[\frac{5}{\lambda}+\sqrt{\lambda(1-\widetilde{\sigma})}\right]+\widetilde{\sigma}\right\} \\
& \leqq \frac{9 \theta}{4 \lambda}
\end{aligned}
$$

The other terms can be bounded in a similar manner giving

$$
\left\|\Delta^{2} B\right\| \leqq 9 \frac{\theta}{\lambda}+\frac{16}{\lambda} \widetilde{\sigma}^{\nu+2} \leqq \frac{5.4}{\lambda} .
$$

Finally the following result holds.

Proposition 3. If $\lambda \geqq 3$ and $\alpha \leqq 1 / 4$ then

$$
\|Q-B\| \leqq(2.7) h \exp \left[2 h \lambda a^{2}\right] a^{2}
$$

with $h \leqq 32 / 3$.

It is possible to obtain bounds on the third difference $\left\|\Delta^{3} B\right\|$ by similar procedures. The algebra becomes somewhat more cumbersome. Nevertheless, it is not difficult to see that bounds of the type

$$
\|Q-B\| \leqq C \frac{\log \lambda}{\sqrt{\bar{\lambda}}} \exp \left[2 \lambda a^{2} h\right] a^{2}
$$

can be obtained in this manner.

The bounds given in Propositions 2 and 3 will be of value if $\lambda a^{2}$ is small. When $\lambda$ is so large that $\lambda \alpha^{2}$ is large, better inequalities than the preceding may be obtained through the use of Fourier transforms. Let $\hat{\mu}$ be the Fourier transform of the measure $\mu$. For instance $\hat{Q}(t)=\int e^{i t x} Q(d x)$. Note the following inequalities.

First

$$
\left|1+p\left(e^{i t}-1\right)\right|^{2}=1-2 p(1-p)(1-\cos t) .
$$

Hence, if $|t| \geqq \pi / 2$ 


$$
\left|1+p\left(e^{i t}-1\right)\right|^{2} \leqq 1-2 p(1-p) \frac{2}{\pi}|t|
$$

If $|t| \leqq \pi / 2$ then

$$
1-\cos t=\frac{t^{2}}{2}\left[1-\frac{t^{4}}{12} \cos \xi t\right]
$$

with $|\xi| \leqq 1$.

Consequently, for $|t| \leqq \pi / 2$

$$
\left|1+p\left(e^{i t}-1\right)\right|^{2} \leqq 1-2 p(1-p) \frac{t^{2}}{2}\left(\frac{48-\pi^{2}}{48}\right)
$$

and for $|t| \leqq \pi / 4$

$$
\left|1+p\left(e^{i t}-1\right)\right|^{2} \leqq 1-2 p(1-p) \frac{t^{2}}{2}\left(\frac{192-\pi^{2}}{192}\right) .
$$

It follows that $|\hat{B}(t)| \leqq 1$ and

(1) For $\pi / 2 \leqq|t| \leqq \pi$ $\max \{|\hat{B}(t)|,|\hat{Q}(t)|\} \leqq \exp -\{\lambda(1-\widetilde{\sigma})(2 / \pi)|t|\}$.

(2) For $\pi / 4 \leqq|t| \leqq \pi / 2$ $\max \{|\hat{B(t)}|,|\hat{Q}(t)|\} \leqq \exp \left[-\left(b^{2} / 2\right) \lambda t^{2}\right]$

with $b^{2}=(1-\widetilde{\sigma})-\pi^{2} / 48$.

(3) For $|t| \leqq \pi / 4$

$$
\max \{|\hat{B(t)}|,|\hat{Q}(t)|\} \leqq \exp \left[-\left(\beta^{2} / 2\right) \lambda t^{2}\right]
$$

with $\beta^{2}=(1-\widetilde{\sigma})\left(1-\pi^{2} / 192\right)$.

In addition, for $|t| \leqq \pi / 4$ and for $z=e^{i t}-1$ one can write

$$
\begin{aligned}
& \log \hat{Q}-\log \hat{B}=\lambda \Sigma c_{j}\left[\frac{1}{p_{j}} \log \left(1+p_{j} z\right)-\frac{1}{\widetilde{\sigma}} \log (1+\widetilde{\sigma})\right] \\
& =-\lambda z^{3} \int_{0}^{1} \frac{\xi^{2}}{(1+\xi \widetilde{\sigma} z)^{2}}\left[\sum_{j} \frac{c_{j} \delta_{j}^{2}}{1+\xi p_{j} z}\right] d \xi
\end{aligned}
$$

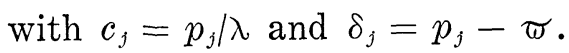

This gives

$$
|\log \hat{Q}-\log \hat{B}| \leqq \frac{1}{3} \lambda \alpha^{2}|z|^{3} \psi(z)
$$

where

$$
\psi(z)=\sup _{|t| \leqq \pi / 4} \sup _{j}\left|\int_{0}^{1} \frac{3 \xi^{2}}{[1+\xi \omega z]^{2}} \frac{1}{\left(1+\xi p_{j} z\right)}\right| d \xi
$$


Since

$$
\begin{aligned}
|1+\xi \varpi z|^{2} & =\left|(1-\xi \varpi)+\xi \varpi e^{i t}\right|^{2} \\
& =1-2 \xi \varpi(1-\xi \varpi)(1-\cos t)
\end{aligned}
$$

one has

$$
|1+\xi \varpi z|^{2} \geqq 1-(2-\sqrt{2}) \frac{\pi}{4} \widetilde{\sigma} .
$$

Finally

$$
\psi(z) \leqq \frac{1}{\sqrt{1-\frac{\alpha}{2}}} \frac{1}{\left(1-\frac{\sigma}{2}\right)}
$$

Hence

$$
|\log \hat{Q}-\log \hat{B}| \leqq K^{2} \lambda a^{2}|t|^{3}
$$

with

$$
K^{2} \leqq \frac{1}{3}\left(1-\frac{\widetilde{\sigma}}{2}\right)^{-1}\left(1-\frac{\alpha}{2}\right)^{-1 / 2}
$$

It follows that, for $|t| \leqq \pi / 4$ one can write

$$
\begin{aligned}
|\hat{Q}(t)-\hat{B}(t)| & \leqq|\hat{B}(t)| \lambda a^{2} K^{2}|t|^{3} \exp \left[\lambda a K^{2}|t|^{3}\right] \\
& \leqq \lambda a^{2} K^{2}|t|^{3} \exp \left[-\frac{1}{2} \lambda \gamma^{2} t^{2}\right]
\end{aligned}
$$

with $\gamma^{2}=\beta^{2}-a^{2} K^{2} \pi / 4 \geqq 0$.

Let $V=(Q-B)$. The individual terms of $V$ are given by the formula

$$
V(k)=\frac{1}{2 \pi} \int_{-\pi}^{+\pi} e^{-i k t} \hat{V}(t) d t .
$$

Applying to this formula the above inequalities one obtains:

$$
\begin{aligned}
& 2 \pi|V(k)| \leqq 2 \lambda a^{2} K^{2} \int_{0}^{\infty} t^{3} \sup \left[-\frac{1}{2} \lambda \gamma^{2} t^{2}\right] d t \\
& +4 \int_{\pi / 4}^{\infty} \exp \left[-\lambda b^{2} \frac{t^{2}}{2}\right] d t \\
& +4 \int_{\pi / 2}^{\infty} \exp \left[-\lambda(1-\varpi) \frac{2}{\pi}\right] d t \text {. }
\end{aligned}
$$

Therefore,

$2 \pi|V(k)| \leqq \frac{4 K^{2} a^{2}}{\lambda \gamma^{4}}+\frac{16}{\lambda \pi b^{2}} \exp \left[-\frac{\lambda b^{2} \pi^{2}}{32}\right]+\frac{2 \pi}{\lambda(1-\varpi)} \exp [-(1-\widetilde{\sigma}) \lambda]$. 
Noting that $x e^{-x} \leqq e^{-1}$ for $x \geqq 0$, this gives

$$
2 \pi \lambda|V(k)| \leqq \frac{4 K^{2} a^{2}}{\gamma^{4}}+\left\{\frac{16 \times 32}{\pi^{3} c b^{4}}+\frac{2 \pi}{(1-\varpi)^{2} e}\right\} \frac{1}{\lambda}
$$

Let $m$ be an integer such that $m \leqq 2 n \pi<m+1$ with $n-1<\nu \leqq n$. The sum of the first $m$ terms of $|V(k)|$ is inferior to

$$
\frac{1}{\pi}\left\{\frac{4 K^{2} a^{2}}{\gamma^{2}}+\frac{16 \times 32}{\lambda \pi^{3} e b^{4}}+\frac{2 \pi}{\lambda(1-\pi)^{2} e}\right\}\left(1+\frac{1}{\nu}\right) .
$$

From this and Chebyshev's inequality it follows that

$$
\begin{aligned}
\|Q-B\| \leqq & \frac{1}{\pi}\left(1+\frac{1}{\nu}\right)\left\{\frac{4 K^{2} a^{2}}{\gamma^{4}}+\frac{16 \times 32}{\lambda \pi^{3} e b^{4}}+\frac{2 \pi}{\lambda(1-\varpi)^{2} e}\right\} \\
& +\frac{(1-\varpi)}{\lambda}+\frac{1}{\lambda}\left[1+4 \widetilde{\varpi}^{\nu+2}\right] .
\end{aligned}
$$

As a summary, one can state the following.

Proposition 4. Assume $\lambda \geqq 3$ and $\alpha \leqq 1 / 4$. Then, there exist constants $C_{1}$ and $C_{2}$ such that

$$
\|Q-B\| \leqq C_{1} a^{2}+C_{2} \lambda^{-1} .
$$

5. Approximation of the binomial by a Poisson distribution. A theorem of Yu. V. Prohorov [9] states that the binomial $B=[I+\widetilde{\sigma} \Delta]^{2}$ and the Poisson $P=\exp (\lambda \Delta)$ differ little. Explicitly, there is a constant $C_{0}$ such that $\|P-B\| \leqq C_{0} \approx$.

Prohorov's result is proved in [9] only for integer values of $\nu$. For this reason we shall give here a complete proof which happens to be somewhat simpler than Prohorov's original argument. This proof leads to an evaluation of the constant $C_{0}$ which may not be the best available but will serve our purposes.

Let $R(x)$ be the ratio of the binomial probability $B[\{x\}]$ to the Poisson probability $P[\{x\}]$

$$
R(x)=\nu(\nu-1) \cdots(\nu-x+1) \widetilde{\sigma}^{x}(1-\widetilde{\sigma})^{\nu-x} e^{\lambda} \lambda^{-2} .
$$

Let us restrict ourselves to the interval $0 \leqq x \leqq n$. Since

$$
\frac{R(x+1)}{R(x)}=\frac{\nu-x}{\nu(1-\varpi)}
$$

the ratio $R$ achieves in this interval a maximum at the point $x$ such that $x-1 \leqq \lambda<x$.

For this particular value of $x$, Stirling's formula leads to the inequality 


$$
\log R(x) \leqq-\frac{1}{2} \log (1-\xi)
$$

with

$$
\widetilde{\sigma}<\xi \leqq \approx\left(1+\frac{1}{\lambda}\right)
$$

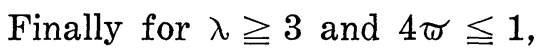

$$
\begin{aligned}
& R(x) \leqq \frac{1}{\sqrt{1-\xi}} \leqq 1+\frac{\xi}{2 \sqrt{1-\xi}}
\end{aligned}
$$

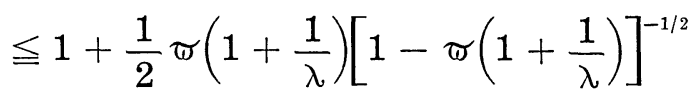

$$
\begin{aligned}
& \leqq 1+\left(\frac{2}{3}\right)^{1 / 2} \pi \text {. }
\end{aligned}
$$

Let $f$ be a nonnegative function such that $0 \leqq f \leqq 1$. The above inequalities imply that

$$
\begin{aligned}
& \int f d B \leqq \frac{4 \widetilde{\sigma}^{\nu+1}}{\nu+1}+\int_{x \leqq n} R(x) f(x) P(d x) \\
& \leqq \frac{4 \widetilde{\sigma}^{\nu+1}}{\nu+1}+\left(\frac{2}{3}\right)^{1 / 2} \varpi \int f(x) P(d x)+\int f(x) P(d x) \\
& \leqq \int f(x) P(d x)+\widetilde{\sigma}\left\{\left(\frac{2}{3}\right)^{1 / 2} \frac{4 \widetilde{\sigma}^{2}}{\nu+1}\right\} \text {. }
\end{aligned}
$$

Similarly,

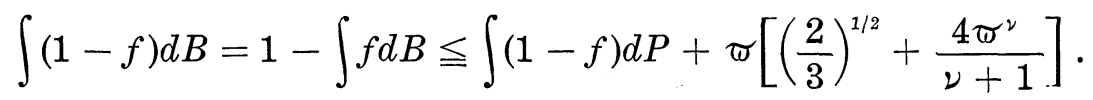

Consequently:

Proposition 5. If $\lambda \geqq 3$ and $4 \varpi \leqq 1$, then

$$
\begin{aligned}
& \|B-P\| \leqq 2 \varpi\left[\left(\frac{2}{3}\right)^{1 / 2}+\frac{4 \varpi^{\nu}}{\nu+1}\right] \\
& \leqq[1.64] \sigma \text {. }
\end{aligned}
$$

Collecting the inequalities established in the preceding sections one obtains the following statement.

Theorem 2. Let $\left\{X_{j} ; j=1,2, \cdots\right\}$ be a family of independent random variables. Assume that $\mathscr{L}\left(X_{j}\right)=I+p_{j} \Delta$ and that $\lambda=\Sigma p_{i}$ is 
finite. Let $p_{j}=\lambda c_{j}$ and $\widetilde{\sigma}=\Sigma c_{j} p_{j}$ and $\alpha=\sup _{j} p_{j}$. Denote by $Q$ the distribution $Q=\mathscr{L}\left(\Sigma X_{j}\right)$ and $P$ the Poisson distribution $P=\exp (\lambda \Delta)$.

There exist constants $D_{1}$ and $D_{2}$ such that

(1) For all values of the $p_{j}$ one has

$$
\|P-Q\| \leqq 2 \lambda \approx
$$

and

$$
\|P-Q\| \leqq D_{1} \alpha
$$

(2) If $4 \alpha \leqq 1$ then

$$
\|P-Q\| \leqq D_{2} \widetilde{\sigma}
$$

The constant $D_{1}$ is inferior to 9 and the constant $D_{2}$ is inferior to 16.

Proof. The proof of Theorem 2 consists essentially of an evaluation of the constants involved in the bounds given by Propositions 2, 3 and 4. To these propositions one must add the following remarks.

The quantity $a^{2}=\Sigma c_{j}\left(p_{j}-\widetilde{\sigma}\right)^{2}$ can be written

$$
a^{2}=\Sigma c_{j}\left(p_{j}-\frac{\alpha}{2}\right)^{2}-\left(\frac{\alpha}{2}-\approx\right)^{2} .
$$

Hence

$$
a^{2} \leqq \alpha \widetilde{\sigma}\left(1-\frac{\widetilde{\sigma}}{2}\right) \leqq\left(\frac{\alpha}{2}\right)^{2}
$$

In particular $a^{2} \leqq \alpha \widetilde{\sigma}$ and $a \leqq \alpha / 2 \leqq 1 / 8$ for $\alpha \leqq 1 / 4$. The bound $\|Q-P\| \leqq D_{1} \alpha$ is operative only when $D \alpha \leqq 2$. It is therefore sufficient to prove that $\|Q-P\| \leqq D_{1} \alpha$ for $\alpha \leqq 2 D_{1}^{-1}$ and $2 \lambda \geqq D_{1}$. A constant $D_{1}$ can then be obtained through application of Proposition 2 for $\lambda a^{2} \leqq$ $y^{2}$ and Proposition 4 for $\lambda a^{2} \geqq y^{2}$, the quantity $y^{2}$ being adjusted to give the best value available.

Similarly, the second inequality can be proved by use of Propositions 3 and 4 , assuming $2 \lambda \geqq 16$ and $\widetilde{1} \leqq 1 / 8$.

Note that the constants 9 and 16 are certainly much too large. For very small values of $\alpha$ or $\widetilde{\sigma}$ one can obtain much better values of $D_{1}$ and $D_{2}$.

Statement 2 of Theorem 2 implies that the approximation by a Poisson distribution will be good even though a few of the probabilities $P_{\text {, }}$ may be close to the bound $\alpha \leqq 1 / 4$. This will happen provided only that these large values contribute relatively little to the value of $\lambda$, the bulk of $\lambda$ being due to very small values of the $p_{j}$. 


\section{Concluding remarks.}

REMARK 1. It would be highly desirable for the applications to lower the values of the coefficients $D_{1}$ and $D_{2}$ to a more reasonable level. When $\alpha$ is fixed, this can be achieved for $D_{2}$ by restricting the range of values of $\widetilde{w}$ to which the inequalities apply. For instance, taking $4 \alpha=1$ but $\widetilde{\sigma}=10^{-2}$, the coefficient $D_{2}$ can be taken approximately equal to 8 . Such a value being still too large one may inquire whether there is a lower bound to the acceptable values of $D_{2}$.

In this connection the following remarks may be of interest. When $\lambda$ becomes very large the distance $(1 / \widetilde{\sigma})\|Q-B\|$ becomes rapidly negligible. This can be seen for instance by using the inequalities which led to Proposition 4 and the bounds in $a^{2} \log \lambda / \sqrt{\lambda}$ obtained through the use of third differences.

The main contribution to $(1 / \widetilde{\sigma})\|-P\|$ is then attributable to the difference between the binomial $B$ and the Poisson measure $P$.

Prohorov's theorem implies that $(1 / \widetilde{\sigma})\|B-P\|$ cannot be much smaller than (.483). Therefore, one cannot expect to obtain a result of the type $\|Q-P\| \leqq D_{2} \widetilde{\sigma}$ where $D_{2}$ would be substantially smaller than $1 / 2$.

REMARK 2. The result of Theorem 1 cannot be materially improved unless one is willing to restrict further the measures $M_{j}$ or the group $\mathfrak{X}$.

A slight modification of the proof given here leads to the inequality

$$
\|Q-P\| \leqq 2\left[1-\prod_{j}\left(1-\beta_{j}\right)\right],
$$

where $\beta_{j}$ is taken equal to $p_{j}\left(1-e^{-p_{j}}\right)$. The bound so obtained is actually reached for certain choices of the measures $M_{j}$. An example of this can be constructed when $\mathfrak{X}$ is the real line. It is sufficient to take $M_{j}$ to be the probability measure giving all its mass to a point $x_{j}$ and select the values $\left\{x_{j} ; j=1,2, \cdots\right\}$ to be rationally independent. For any fixed $\varepsilon>0$ one may find values $p_{j}<\varepsilon$ such that $2\left[1-\Pi\left(1-\beta_{j}\right)\right]>$ $2-\varepsilon$ and such that $\lambda=\sum_{j} p_{j}$ be finite.

\section{REFERENCES}

1. W. Doeblin, Sur les sommes d'un grand nombre de variables aléatoires indépendantes, Bull. des Sciences Mathématiques, 53, Paris (1939), 23-32.

2. Nelson Dunford and Jacob T. Schwartz, Linear operators, Part I. General theory, Interscience Publishers, New York, 1958.

3. Einar Hille and Ralph S. Phillips, Functional analysis and semi groups, Amer. Math. Soc. Coll. Publ. 31, Providence, R. I. 1957.

4. J. L. Hodges, Jr. and Lucien Le Cam, The Poisson approximation to the Poisson 
binomial distribution, to be published in Ann. Math. Stat.

5. A. Khintchine, Asymptotische Gesetze der Wahrscheinlichkeitsrechnung, Ergebnisse der Mathematik und ihrer grenzgebiete, Julius Springer, Berlin, 1933.

6. A. N. Kolmogorov, Deux théorèmes asymptotiques pour les sommes de variables aléatoires (Russian, French summary), Teoriia Veroiatnosteii, 1 (4), Moscow (1956), 426-436.

7. Paul Lévy, Théorie de l'addition des variables aléatoires, Gauthier-Villars, Paris, 1937.

8. M. A. Naimark, Normed rings, Moscow, 1956.

9. Yu. V. Prohorov, Asymptotic behavior of the binomial distribution (Russian), Uspekhii Matematicheskiikh Nauk, 8 (3), Moscow (1953), 135-142. 



\section{PACIFIC JOURNAL OF MATHEMATICS}

\section{EDITORS}

David GILbarg

Stanford University

Stanford, California

\section{F. H. BRowneLL}

University of Washington

Seattle 5, Washington

\section{A. L. Whiteman}

University of Southern California Los Angeles 7, California

\section{J. PAIGe}

University of California

Los Angeles 24, California

\section{ASSOCIATE EDITORS}
E. F. BECKENBACH
T. M. CHERRY
D. DERRY

\author{
E. HEWITT \\ A. HORN \\ L. NACHBIN
}

\author{
M. OHTSUKA \\ H. L. ROYDEN \\ M. M. SCHIFFER
}

E. SPANIER

E. G. STRAUS

F. WOLF

\section{SUPPORTING INSTITUTIONS}

\author{
UNIVERSITY OF BRITISH COLUMBIA \\ CALIFORNIA INSTITUTE OF TECHNOLOGY \\ UNIVERSITY OF CALIFORNIA \\ MONTANA STATE UNIVERSITY \\ UNIVERSITY OF NEVADA \\ NEW MEXICO STATE UNIVERSITY \\ OREGON STATE COLLEGE \\ UNIVERSITY OF OREGON \\ OSAKA UNIVERSITY \\ UNIVERSITY OF SOUTHERN CALIFORNIA
}

\author{
STANFORD UNIVERSITY \\ UNIVERSITY OF TOKYO \\ UNIVERSITY OF UTAH \\ WASHINGTON STATE COLLEGE \\ UNIVERSITY OF WASHINGTON \\ AMERICAN MATHEMATICAL SOCIETY \\ CALIFORNIA RESEARCH CORPORATION \\ HUGHES AIRCRAFT COMPANY \\ SPACE TECHNOLOGY LABORATORIES \\ NAVAL ORDNANCE TEST STATION
}

\footnotetext{
Mathematical papers intended for publication in the Pacific Journal of Mathematics should be typewritten (double spaced), and the author should keep a complete copy. Manuscripts may be sent to any one of the four editors. All other communications to the editors should be addressed to the managing editor, L. J. Paige at the University of California, Los Angeles 24, California.

50 reprints per author of each article are furnished free of charge; additional copies may be obtained at cost in multiples of 50 .
}

The Pacific Journal of Mathematics is published quarterly, in March, June, September, and December. The price per volume (4 numbers) is $\$ 12.00$; single issues, $\$ 3.50$. Back numbers are available. Special price to individual faculty members of supporting institutions and to individual members of the American Mathematical Society: $\$ 4.00$ per volume; single issues, $\$ 1.25$.

Subscriptions, orders for back numbers, and changes of address should be sent to Pacific Journal of Mathematics, 2120 Oxford Street, Berkeley 4, California.

Printed at Kokusai Bunken Insatsusha (International Academic Printing Co., Ltd.), No. 6, 2-chome, Fujimi-cho, Chiyoda-ku, Tokyo, Japan.

PUBLISHED BY PACIFIC JOURNAL OF MATHEMATICS, A NON-PROFIT CORPORATION

The Supporting Institutions listed above contribute to the cost of publication of this Journal, but they are not owners or publishers and have no responsibility for its content or policies. 


\section{Pacific Journal of Mathematics}

\section{Vol. 10, No. $4 \quad$ December, 1960}

M. Altman, An optimum cubically convergent iterative method of inverting a linear bounded operator in Hilbert space . . . . . . . . . . . . . . . . . . . . . . . . . . 1107

Nesmith Cornett Ankeny, Criterion for rth power residuacity ................. 1115

Julius Rubin Blum and David Lee Hanson, On invariant probability measures I . . . . . 1125

Frank Featherstone Bonsall, Positive operators compact in an auxiliary topology ..... 1131

Billy Joe Boyer, Summability of derived conjugate series . . . . . . . . . . . . . . . . 1139

Delmar L. Boyer, A note on a problem of Fuchs . . . . . . . . . . . . . . . . . 1147

Hans-Joachim Bremermann, The envelopes of holomorphy of tube domains in infinite

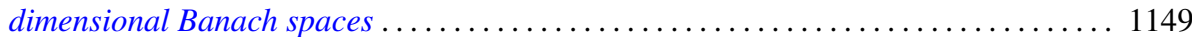

Andrew Michael Bruckner, Minimal superadditive extensions of superadditive

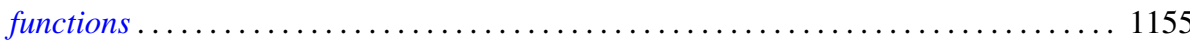

Billy Finney Bryant, On expansive homeomorphisms .................... 1163

Jean W. Butler, On complete and independent sets of operations in finite algebras . . . . . 1169

Lucien Le Cam, An approximation theorem for the Poisson binomial distribution ...... 1181

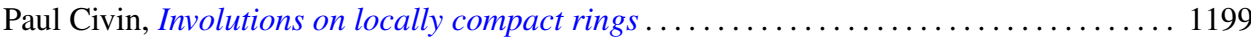

Earl A. Coddington, Normal extensions of formally normal operators . . . . . . . . . 1203

Jacob Feldman, Some classes of equivalent Gaussian processes on an interval ........ 1211

Shaul Foguel, Weak and strong convergence for Markov processes . . . . . . . . . . . 1221

Martin Fox, Some zero sum two-person games with moves in the unit interval ........ 1235

Robert Pertsch Gilbert, Singularities of three-dimensional harmonic functions . . . . . . . 1243

Branko Grünbaum, Partitions of mass-distributions and of convex bodies by

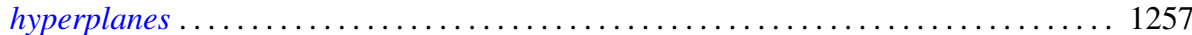

Sidney Morris Harmon, Regular covering surfaces of Riemann surfaces ........... 1263

Edwin Hewitt and Herbert S. Zuckerman, The multiplicative semigroup of integers

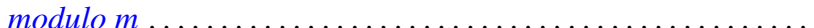

Paul Daniel Hill, Relation of a direct limit group to associated vector groups . ......... 1309

Calvin Virgil Holmes, Commutator groups of monomial groups . .

James Fredrik Jakobsen and W. R. Utz, The non-existence of expansive homeomorphisms

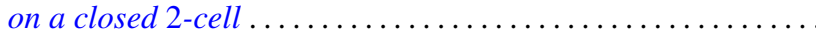

John William Jewett, Multiplication on classes of pseudo-analytic functions . . . . . . . 1323

Helmut Klingen, Analytic automorphisms of bounded symmetric complex domains . . . . 1327

Robert Jacob Koch, Ordered semigroups in partially ordered semigroups . . . . . . . . 1333

Marvin David Marcus and N. A. Khan, On a commutator result of Taussky and

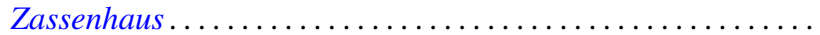

John Glen Marica and Steve Jerome Bryant, Unary algebras......

Edward Peter Merkes and W. T. Scott, On univalence of a continued fraction . . . . . . . 1361

Shu-Teh Chen Moy, Asymptotic properties of derivatives of stationary measures . . . . . 1371

John William Neuberger, Concerning boundary value problems . . . . . . . . . . . 1385

Edward C. Posner, Integral closure of differential rings . . . . . . . . . . . . . . . . . 1393

Marian Reichaw-Reichbach, Some theorems on mappings onto . . . . . . . . . . . . . 1397

Marvin Rosenblum and Harold Widom, Two extremal problems . . . . . . . . . . . . . . . . 1409

Morton Lincoln Slater and Herbert S. Wilf, A class of linear differential-difference

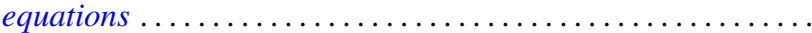

Charles Robson Storey, Jr., The structure of threads . . . . . . . . . . . . . . . . . . 1429

J. François Treves, An estimate for differential polynomials in $\partial / \partial z_{1},, \cdots, \partial / \partial z_{-} n \ldots \ldots 1447$

J. D. Weston, On the representation of operators by convolutions integrals . . . . . . . . 1453

James Victor Whittaker, Normal subgroups of some homeomorphism groups ......... 1469 